\title{
New aspects of the electrochemical-catalytic (EC') mechanism in square-wave voltammetry
}

\author{
Rubin Gulaboski ${ }^{\mathrm{a}, \mathrm{b}, *}$, Valentin Mirceski ${ }^{\mathrm{a}, \mathrm{b}}$ \\ a Faculty of Medical Sciences, Goce Delcev University, Stip, Macedonia \\ ${ }^{\mathrm{b}}$ Institute of Chemistry, Faculty of Natural Sciences and Mathematics, “Ss Kiril i Metodij” University, Skopje, Macedonia
}

\section{A R T I C L E I N F O}

\section{Article history:}

Received 19 January 2015

Received in revised form 20 March 2015

Accepted 24 March 2015

Available online 27 March 2015

\section{Keywords:}

EC' catalytic mechanism

electrode reaction kinetics

standard rate constant

enzymatic biosensors

protein-film voltammetry

\begin{abstract}
A B S T R A C T
Several new theoretical aspects of the electrocatalytic (regenerative) EC' mechanism under conditions of square-wave (SWV) and staircase cyclic voltammetry (SCV) are presented. Elaborating the effect of the rate of the catalytic reaction in the diffusion-controlled catalytic mechanism (diffusional EC' mechanism) and surface catalytic mechanism (surface EC' mechanism), we refer to several phenomena related to the shift of the position and the half-peak width of the net peak in square-wave voltammetry (SWV). If the rate of the catalytic reaction is much higher than the kinetics of the electrode reaction, a linear dependence between the peak potential of the simulated net SWV peaks and the logarithm of the catalytic parameter can be observed. The intercept of that linear dependence is a function of the kinetics of the electrode reaction. Based on this finding, we propose a new methodology to determine the electrode kinetics rate constant. The proposed approach relies on the variation of the concentration of the regenerative reagent. To the best of our knowledge, this is one of very few voltammetric approaches for electrode kinetic measurements not based on the time or potential variation in the experimental analyzes. In addition, we present a brief analysis of the catalytic mechanism under conditions of staircase cyclic voltammetry in order to emphasize the main differences between SCV and SWV.
\end{abstract}

(c) 2015 Elsevier Ltd. All rights reserved.

\section{Introduction}

Electron transfer reactions that are coupled with preceding or follow-up chemical reactions are extensively studied in the last 40 years. A lot of theoretical features of the EC (electrochemicalchemical), CE (chemical-electrochemical), ECE (electrochemicalchemical-electrochemical), EC' (electrochemical-catalytic) systems under voltammetric conditions at various electrodes are appropriately elaborated in several excellent books [1-6], theoretical studies and reviews [7-26]. The so-called electrocatalytic regenerative mechanism (often referred to as EC' or ECat mechanism) is one of the most intriguing from a theoretical point of view, since it differs in many aspects from the other three mechanisms (EC, CE, and ECE) $[1,5-8,11,13,16,18-26]$. The EC' electrode mechanism comprises regeneration of the initial electroactive species involved in the electrode reaction via homogeneous redox reaction of the electrochemically generated product with other redox species present in the electrolyte solution. The latter species are commonly electrochemically inactive in the considered potential window. A common scheme that represents the EC'

\footnotetext{
* Corresponding author. Tel.: +389 78210883.

E-mail address: rubin.gulaboski@ugd.edu.mk (R. Gulaboski).
}

mechanism is:

$$
\begin{aligned}
\text { EOx }+ \text { ne }- & \rightleftarrows{ }^{\mathrm{ks}} \text { Red } \\
& \mathrm{C}^{\prime} \text { Red }+\mathrm{Y} \rightarrow{ }^{\mathrm{kc}} \mathrm{Ox}(+ \text { side electroinactive products })
\end{aligned}
$$

The chemical reaction ( $\left.C^{\prime}\right)$ between Red and Y ( $\mathrm{Y}$ is named a "regenerative" reagent) resupplies additional amount of the initial electroactive material $(\mathrm{Ox})$ that can undergo multiple electron transfer at the working electrode. This phenomenon leads to an increase of the measured current as a consequence of the multiple reuse of the starting electroactive material $(\mathrm{Ox})$ during the timeframe of the voltammetric experiment. Since the current enhancement is a major factor for achieving a better sensitivity of a given electroanalytical method, this unique feature of the EC' mechanism is explored for designing numerous sensors used for quantification of many important molecules and enzymes [1,7,8,27-29]. Of these, the so-called "voltammetric enzymatic biosensors" are probably the most important [27-29]. Although the EC' electrode mechanism is one of the most thoroughly studied under voltammetric conditions [1,8,16-34], we give in this work some new insights that are not elaborated so far in detail. The main focus of this theoretical work is on the features of the square-wave (SW) voltammograms of a quasireversible electrode reaction 
coupled with a chemical catalytic reaction. The theoretical analysis is conducted under conditions of significantly higher rate of the catalytic redox reaction (Red $+Y$ ), compared to the rate of the electrode reaction ( $\mathrm{Ox}+$ ne $\rightarrow$ Red). This affects the concentration gradients of the electroactive species and the apparent reversibility of the electrode reaction. Hence, a simple and time-independent method for estimation of the electrode reaction rate can be developed, on the basis of altering the rate of the catalytic reaction via changing the concentration of the catalytic reagent $Y$ only. The theoretical analysis encompasses EC' mechanism of surface confined species $[16,17]$ as well as a mechanism involving solution resident species where the mass transfer phenomena are controlled by diffusion [1].

The theoretical analysis takes an advantage of a comparison between square-wave (SWV) and the staircase cyclic voltammetry (SCV) in order to give hints about the causes leading to differences in the response of the two techniques, as well as to understand the behaviour of the system under complex conditions of SWV. It is worth noting that in a modern digital electrochemical instrumentation, the staircase form of cyclic voltammetry is currently one of the most frequently used potentiodynamic technique for inspections of electrochemically active compounds. The response of SCV differs from classical linear sweep cyclic voltammetry, thus a careful analysis of simulated data considered under conditions of SCV is reasonable even for well-known electrode reaction pathways, i.e., the EC' mechanism. In a general context, cyclic voltammetry, either with a linear sweep or a staircase ramp, is the most frequently used technique for mechanistic studies of chemical reaction coupled electrode mechanisms [18,35,36], which is based on a mechanism specific morphological evolution of the voltammetric response. From the features of the cyclic voltammograms as a function of the scan rate, one can both qualitatively and quantitatively characterize particular electrode mechanism [18-26,34,35]. In the last 30 years, however, squarewave voltammetry emerged as a fast and powerful technique that offers many possibilities for qualitative characterization of various electrode mechanisms, providing at the same time reliable and simple methods for thermodynamic and kinetic measurements [1].

\section{Theoretical aspects}

We consider theoretically two EC' electrode mechanisms that can be represented by the following schemes:

(A)

$\mathrm{EOx}+\mathrm{ne}-\rightleftarrows^{\mathrm{ks}} \mathrm{Red}$

$\mathrm{C}^{\prime} \mathrm{Red}+\mathrm{Y} \rightarrow{ }^{\mathrm{kc}} \mathrm{Ox}(+$ side electroinactive products $)$

$\mathrm{EOx}(\mathrm{ads})+\mathrm{ne}-\rightleftarrows^{\mathrm{ksur}} \operatorname{Red}(\mathrm{ads})$

$\mathrm{C}^{\prime} \operatorname{Red}(\mathrm{ads})+\mathrm{Y} \stackrel{\mathrm{kc}}{\rightarrow} \mathrm{Ox}(\mathrm{ads})(+$ side electroinactive products $)$

The considered mechanism (A) is named as a "diffusional EC' electrode mechanism", where all redox active participants are dissolved in the electrolyte solution. The mechanism (B) is socalled "surface EC' electrode mechanism", in which both electroactive species (Ox and Red) are firmly adsorbed (immobilized) on the electrode surface.

Mathematically, the diffusional electrode mechanism (A) can be described by the following set of differential equations [1]:

$[\mathrm{d} c(\mathrm{Ox}) / \mathrm{d} t]=D\left[\mathrm{~d}^{2} c(\mathrm{Ox}) / \mathrm{d} x^{2}\right]+c(\operatorname{Red}) k_{\mathrm{c}}$ $[\mathrm{d} c(\operatorname{Red}) / \mathrm{d} t]=D\left[\mathrm{~d}^{2} c(\operatorname{Red}) / \mathrm{d} x^{2}\right]-c(\operatorname{Red}) k_{\mathrm{c}}$

The differential Eqs. (1)-(2) are solved under the following boundary conditions:

(a) $t=0, x \geq 0, c(\mathrm{Ox})=c^{*}(\mathrm{Ox}), c($ Red $)=0$;

(b) $t>0, x \rightarrow \infty, c(\mathrm{Ox}) \rightarrow c^{*}(\mathrm{Ox}), c($ Red $) \rightarrow 0$;

(c) $t>0, x=0$ :

$D[\partial c(\mathrm{Ox}) / \partial x]_{\mathrm{x}=0}=-D[\partial c(\operatorname{Red}) / \partial x]_{\mathrm{x}=0}=[I /(n F S)] ;$ and

$I /(n F S)=k_{S} \exp (-\alpha \varphi)\left[c(\mathrm{Ox})_{\mathrm{x}=0}-\exp (\varphi) c(\operatorname{Red})_{\mathrm{x}=0}\right]$

Here, $t$ is time (s); $x$ is distance from the electrode surface $(\mathrm{cm})$; $c$ is concentration and $c^{*}$ is bulk concentration; $D$ is the common diffusion coefficient $\left(\mathrm{cm}^{2} \mathrm{~s}^{-1}\right) ; k_{\mathrm{s}}$ is the standard rate constant of the electrode reaction $\left(\mathrm{cm} \mathrm{s}^{-1}\right) ; I$ is current $(\mathrm{A}) ; n$ is number of electrons exchanged; $\alpha$ is the cathodic (reductive) electron transfer coefficient; $F$ is the Faraday constant $(96,485 \mathrm{C} / \mathrm{mol}) ; S$ is the electrode surface area $\left(\mathrm{cm}^{2}\right) ; \varphi=n F / R T\left(E-E^{0}\right)$ is dimensionless potential where $E$ is electrode potential and $E^{0}$ is standard potential of the electrode reaction; $R$ is the gas constant $\left(8.314 \mathrm{~J} \mathrm{~mol}^{-1} \mathrm{~K}^{-1}\right)$, and $T$ is the thermodynamic temperature $(\mathrm{K})$. Note that $k_{\mathrm{c}}$ is a pseudo-first-order "catalytic" rate constant of the reaction Red + $\mathrm{Y} \rightarrow \mathrm{Ox}$. It is defined as $k_{\mathrm{c}}=k_{\mathrm{cat}} \mathrm{c}(\mathrm{Y})$. $k_{\mathrm{cat}}$ is the real rate constant of the catalytic redox reaction in units of $\mathrm{s}^{-1} \mathrm{~mol}^{-1} \mathrm{~L}$, while $c(\mathrm{Y})$ is the molar concentration of the catalyzing (regenerating) agent $\mathrm{Y}$ (usually it is present in a large excess compared to the bulk concentration of $\mathrm{Ox}$ ).

The "surface EC' electrode mechanism" (B) can be mathematically described by the following equations [17]:

$[\mathrm{d} \Gamma(\mathrm{Ox}) / \mathrm{d} t]=-I /(n F S)+\Gamma($ Red $) k_{\mathrm{c}}$

$[\mathrm{d} \Gamma(\operatorname{Red}) / \mathrm{d} t]=I /(n F S)-\Gamma(\operatorname{Red}) k_{\mathrm{c}}$

For the surface EC' mechanism, the differential Eqs. (3)-(4) are solved under following conditions:

- $t=0 ; \Gamma(\mathrm{Ox})=\Gamma^{*}(\mathrm{Ox}) ; \Gamma($ Red $)=0$

- $t>0 ; \Gamma(\mathrm{Ox})+\Gamma(\mathrm{Red})=\Gamma^{*}(\mathrm{Ox})$

- $I /(n F S)=k_{\text {sur }} \exp (-\alpha \varphi)[\Gamma(\mathrm{Ox})-\exp (\varphi) \Gamma($ Red $)]$

$\Gamma^{*}(\mathrm{Ox})$ is initial surface concentration of the starting material Ox adsorbed on the electrode surface $\left(\mathrm{mol} \mathrm{cm}^{-2}\right), \Gamma$ is surface concentration, $k_{\text {sur }}$ is surface standard rate constant of the electrode reaction $\left(\mathrm{s}^{-1}\right)$, and other symbols have the same meaning as for the mechanism $(A)$.

The solutions of the differential Eqs. (1)-(2) and (3)-(4) under conditions of square-wave voltammetry are given elsewhere $[1,8,16,17]$. For both mechanisms the reductive current is assumed to be positive. For the diffusional EC' mechanism, as implied by the mathematical model, we have assumed that the mass transfer of electroactive species takes place via semi-infinite linear diffusion. For the surface EC' mechanism, we assume that the Ox and Red species are firmly adsorbed on the electrode surface, and there are no interactions between the adsorbed species. All simulations have been performed by the commercial package MATHCAD 14 .

\section{Results and discussion}

\subsection{Diffusional EC' mechanism}

The features of the simulated voltammograms are function of the parameters of the potential modulation, i.e., in SCV, the scan 


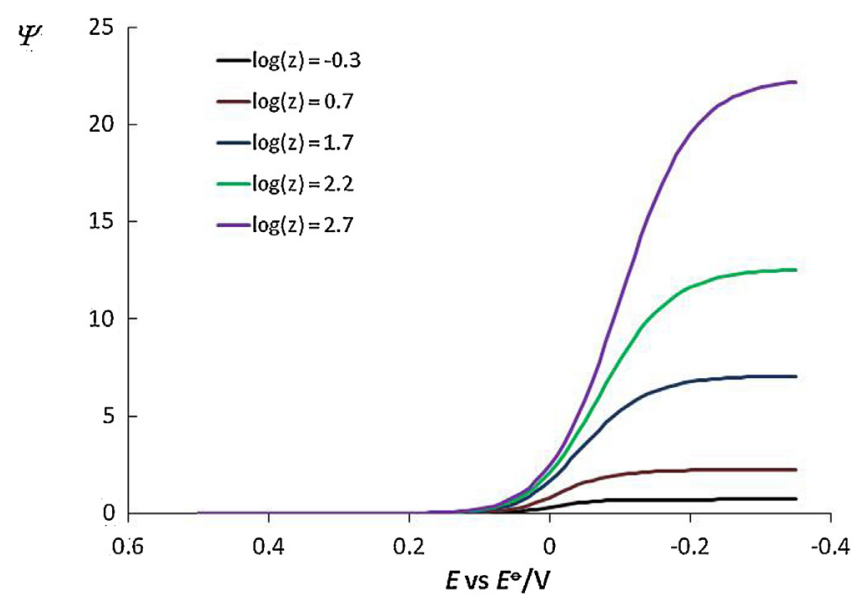

Fig. 1. Effect of the catalytic parameter $z$ on the shape of the staircase cyclic voltammograms of the EC'diffusional mechanism featuring moderate electrode kinetics associated with $\log (K)=0.5$ simulated for the scan rate of $50 \mathrm{mV} / \mathrm{s}$. The values of the catalytic parameter $z$ are given on the plot.

rate defined as $\mathrm{d} E / \tau$, where $\mathrm{d} E$ is the potential step, and $\tau$ is the duration of the potential step, and, in SWV, on the potential step $(\mathrm{d} E)$, frequency $(f)$, and height of the potential pulse (square-wave amplitude- $\left.E_{\mathrm{sw}}\right)$. Additionally, the cathodic electron transfer coefficient $\alpha$, the dimensionless kinetic parameter $\boldsymbol{K}$ (related to the electrode reaction), and the dimensionless "catalytic" parameter $\boldsymbol{z}$ (related to the kinetics of the regenerative reaction), also affect the features of simulated voltammograms. For the diffusional EC' mechanism, the dimensionless kinetic parameter $K$ is defined as $K=k_{\mathrm{s}}(D f)^{-0.5}$ (for SWV) and $K=k_{\mathrm{s}} \tau^{0.5} D^{-0.5}$ (for SCV). The electrode kinetic parameter $K$ represents the apparent electrochemical reversibility of the electrode reaction by comparing the rate of the electron transfer $\left(k_{\mathrm{s}}\right)$ with the rate of the diffusion, i.e., $(D f)^{0.5}$ for SWV and $(D / \tau)^{0.5}$ for SCV. The dimensionless catalytic parameter is defined as $z=k_{\mathrm{c}} / f$ (for SWV) and $z=k_{\mathrm{c}} \tau$ (for SCV), representing the intrinsic influence of the rate of the chemical regenerative reaction $\left(k_{\mathrm{c}}\right)$ relative to the time window of the voltammetric experiment represented by $f$ in SWV and $\tau$ in SCV. The dimensionless currents are defined as $\Psi=I\left[n F S c^{*}\right.$
$(\mathrm{Ox})]^{-1}(f D)^{-0.5}$ and $\Psi=I\left[n F S c^{*}(\mathrm{Ox})\right]^{-1} \tau^{0.5} D^{-0.5}$ for SWV and SCV, respectively.

The effect of the catalytic parameter $z$ to the features of the staircase cyclic voltammograms has been initially studied for an electrode reaction characterized with fast electron transfer (log $(K)=1.4$ ) (data not shown). We note that this is still a quasireversible electrode reaction as the real Nernstian behaviour is expected to be achieved for $\log (K)>2$ [1]. As well known, by increasing the value of the catalytic parameter $\boldsymbol{z}$ the magnitude of the response increases and the shape changes, from a peak-like (diffusion controlled) to a sigmoid one, dictated by the rate of the catalytic reaction. This is the consequence of the diminishing of the reverse and concomitant increase of the direct current component of the staircase cyclic voltammograms. When the catalytic parameter $z$ gets values higher than $0.01(\log (z)>-2)$, a steadystate, sigmoid shaped current response is attained even for electrode reaction characterized with $\log (K)=1.4$, while for $\log$ $(\boldsymbol{z})>-1.3$, the direct and reverse branches of the staircase cyclic voltammogram become identical. Obviously, under such conditions, the rate of the catalytic reaction Red $+\mathrm{Y} \rightarrow \mathrm{Ox}$ is much higher than the rate of both electrode reaction and the diffusion mass transport, providing a permanent amount of the initial electroactive reactant $\mathrm{Ox}$ at the electrode surface. Therefore, the measured currents of the mechanism (A) in both direct and reverse potential direction are actually reductive currents due to the occurrence of the one directional electrode reaction only: $\mathrm{Ox}+n \mathrm{e}^{-} \rightarrow$ Red.

Interestingly, for a slower electrode reaction $(\log (K)=0.5)$ under the influence of a fast regenerative chemical reaction (log $(\boldsymbol{z})>-0.3)$, the half-wave potential of the sigmoid steady state staircase cyclic voltammogram shifts systematically by increasing $\boldsymbol{z}$ (Fig. 1). Shifting of the half-wave potential in a negative potential direction (for a reductive mechanism) in voltammetry implies kinetic hindrances of the electrode reactions, manifested as additional overpotential needed to drive the electrode reaction. In the present mechanism, this phenomenon appears at very high values of the catalytic parameter $\boldsymbol{z}$, i.e., when the concentration ratio of the electroactive species at the electrode surface $c(\mathrm{Ox})_{\mathrm{X}=0} / c$ $(\text { Red })_{x=0}$ is strongly affected. The data from Fig. 1 suggest that the apparent electrochemical reversibility of the overall electrode mechanism is affected by the rate of both the electrode and the
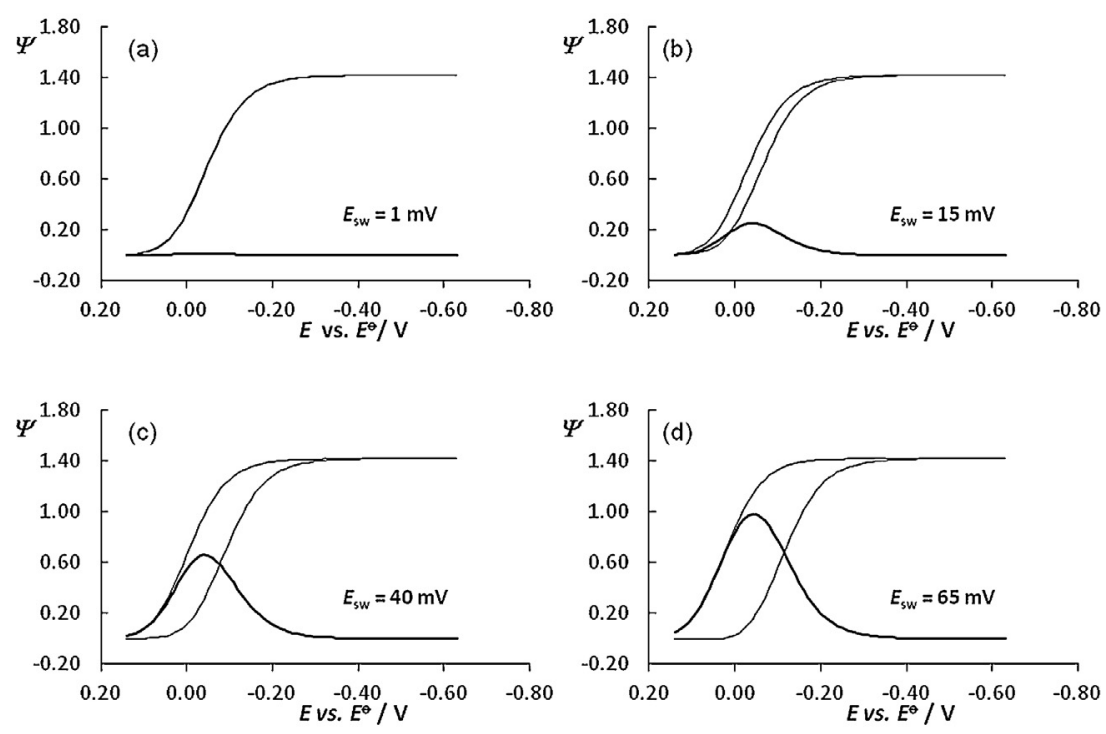

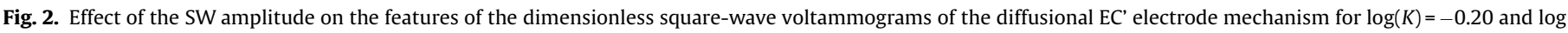

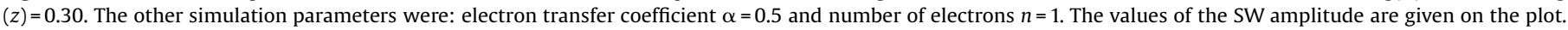



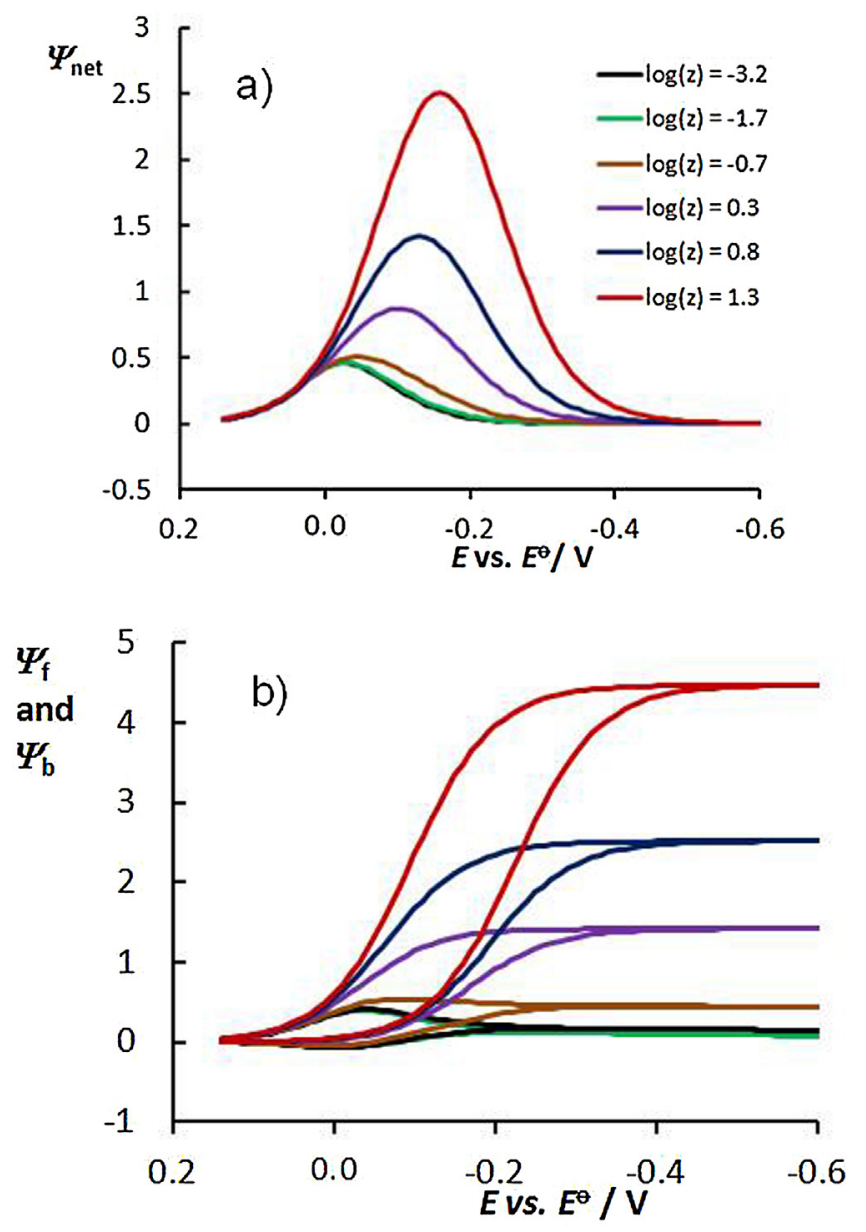

Fig. 3. Effect of the catalytic parameter $z$ on the properties of the simulated net (a) and forward and backward current components (b) of the square-wave voltammograms for the EC' diffusional mechanism featuring quasireversible electrode reaction $\log (K)=-0.70$. All the other simulation parameters were the same as those in Fig. 2. The values of the catalytic parameter $z$ are given on the plot. regenerative chemical reaction, i.e., it depends on both dimensionless parameters $K$ and $z$.

This phenomenon is further analyzed in more detail under conditions of SWV. We recall that a notable feature of the SW voltammograms is the well-defined peak-shape of the net SW peaks for any rate of the electrode and catalytic reaction [1]. For high rates of the catalytic reaction, the forward and backward components of the SW voltammograms get a steady-state shape, similar to the staircase cyclic voltammograms. However, contrary to the staircase cyclic voltammograms, where both the forward and the backward currents are becoming identical for $\boldsymbol{z}>0.05$, in SWV there is a certain measurable current difference between the forward and the backward components for all values of $\boldsymbol{z}$. This, in turn, produces a net SW output in a form of a peak, since the net current in SWV is defined as a difference between the forward and the backward current branches [1]. The cause for observing dissimilarities between the forward and backward current components in SWV and SCV is due to the square-wave amplitude and in specific means of presenting the voltammetric data in SWV [1] (Fig. 2). Following the current sampling procedure in SWV, we recall that the $S W$ amplitude is actually a potential pulse of a given height by which the staircase potential ramp is modified. For example, if an amplitude of $50 \mathrm{mV}$ is applied to a mean staircase potential of $0.00 \mathrm{~V}$, then in $\mathrm{SWV}$ one measures the current twice: once at potential of $+0.05 \mathrm{~V}(0.00 \mathrm{~V}$ increased by the value of the amplitude of $0.05 \mathrm{~V})$, and once at potential of $-0.05 \mathrm{~V}(0.00 \mathrm{~V}$ diminished by the value of the amplitude of $0.05 \mathrm{~V}$ ). In that way, the measured currents in the forward and the backward step differ, since the rate constant of the electrode reaction at potentials of $+0.05 \mathrm{~V}$ and $-0.05 \mathrm{~V}$ is different. On the other hand, both forward and backward currents in SWV are presented versus the potential of $0.00 \mathrm{~V}$, i.e., the potential of the staircase ramp. Hence, as for other mechanisms, the role of the SW amplitude is of critical importance for the EC' mechanism under the influence of the fast catalytic reaction, which is illustrated by the data presented in Fig. 2. As we can see, when the magnitude of the SW amplitude approaches $0 \mathrm{~V}$, then one observes almost identical values of the forward and backward currents of the SW voltammograms and (almost) no net SW peak (Fig. 2a). This situation resembles cyclic voltammograms presented in Fig. 1. However, as the SW amplitude gets bigger values, more pronounced is the difference between the forward and the backward currents, which, in turn, produces higher net SW

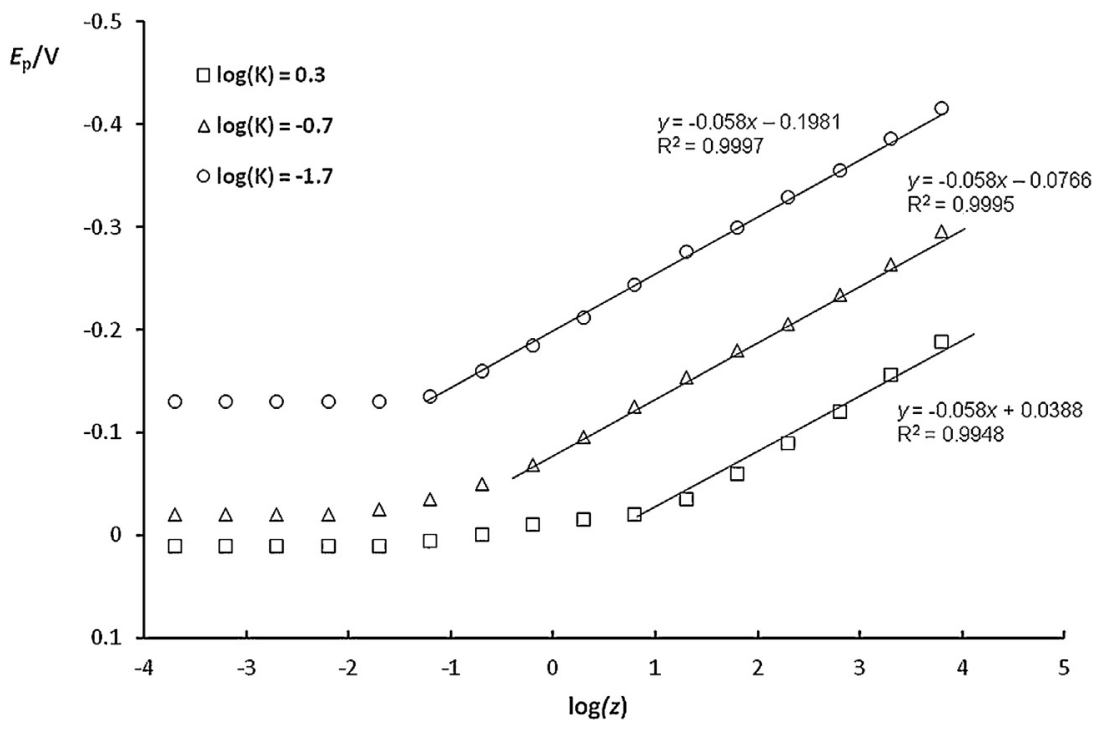

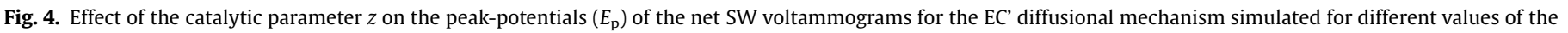
electrode kinetic parameter (values given on the plot). All other simulation parameters were the same as those in Fig. 2 . 


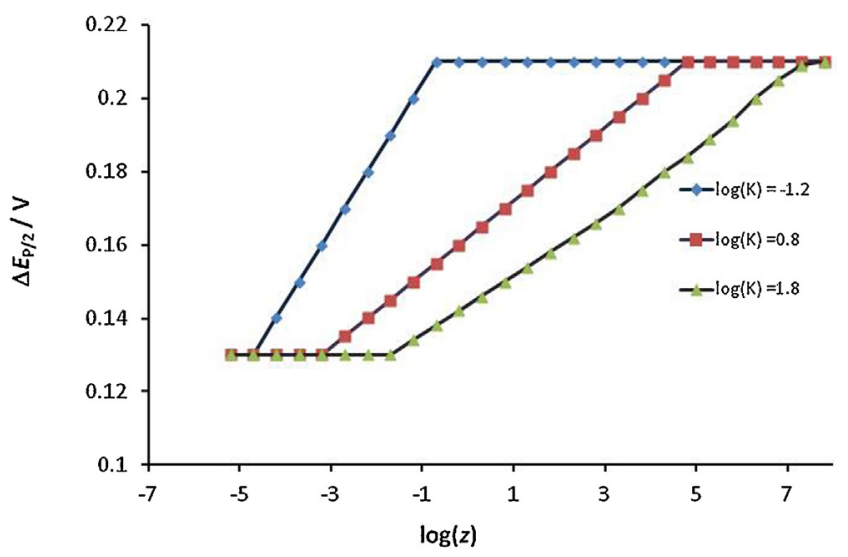

Fig. 5. Effect of the catalytic parameter $z$ on the half-peak widths $\left(\Delta E_{\mathrm{p} / 2}\right)$ of the net SW voltammograms for the EC' diffusional mechanism simulated for different values of the electrode kinetic parameter (values given on the plot). All other simulation parameters were the same as those in Fig. 2.

peaks (Fig. 2 b-d). While the square-wave amplitude affects the dimensionless net peak current and the half-peak width, it is important to stress that the peak potential of the net SW peak remains virtually unaltered. For example, by changing the SW amplitude from $10 \mathrm{mV}$ to $120 \mathrm{mV}$ (for $\log (z)=0.3$ and $\log$ $(K)=-0.2$ and $n=1)$, the peak potential shifts for only $6 \mathrm{mV}$ in positive direction. At the same time, the half-peak widths of the net SW peaks change significantly by increasing the SW amplitude from $130 \mathrm{mV}$ (for $n E_{\mathrm{sw}}=10 \mathrm{mV}$ ) up to $300 \mathrm{mV}$ (for $n E_{\mathrm{sw}}=120 \mathrm{mV}$ ). This, in turn, makes the net SW peak very broad and inappropriate for analysis. Therefore, in order to get square-wave voltammograms suitable for analysis an amplitude value between 25 and $70 \mathrm{mV}$ is recommended.

On the other hand, the position of the net SW peak of a quasireversible electrode reaction is strongly affected if the rate of the catalytic reaction is high (Fig. 3). The latter figure shows squarewave voltammograms featuring moderate, quasireversible, electrode reaction $(\log (K)=-0.7)$, simulated for several values of the catalytic parameter $\boldsymbol{z}$. The increase of the catalytic parameter $\boldsymbol{z}$ from $\log (\boldsymbol{z})=-3.20$ to $\log (\boldsymbol{z})=1.3$, for example, is followed by a shift of the net $\mathrm{SW}$ peak potential $\left(E_{\mathrm{p}}\right)$ for $-58 \mathrm{mV}$ for a ten-fold increase of $\boldsymbol{z}$, considering the reductive mechanism. Note that for an oxidative catalytic mechanism the slope would be identical but with opposite sign. This potential shift depends significantly on the rate of the electrode reaction as depicted in Fig. 4. For all curves presented in Fig. 4, there is a linear dependence between $E_{\mathrm{p}}$ vs. $\log (z)$ with a slope being equal to $-2.303 R T /(n F)$. The intercepts of the $E_{\mathrm{p}} \mathrm{vs} \log (\boldsymbol{z})$ dependences shown in Fig. 4 are defined as: intercept $=\boldsymbol{E}^{0}+\mathbf{2 . 3 0 3 R T}$ $(\boldsymbol{\alpha} \boldsymbol{n} \boldsymbol{F})^{-1} \log (\boldsymbol{K})$, where $\boldsymbol{E}^{0}$ is the standard redox potential of the couple $\mathrm{Ox} /$ Red. The latter equation allows the standard rate constant of electron transfer reaction to be determined, by inspecting the variation of the net SW peak potential as a function of the rate of the catalytic reaction via altering the concentration of catalytic reagent Y. For this, previous knowledge of other parameters such as the standard potential of the redox couple, the number of the electrons exchanged, the electron transfer coefficient, as well as the diffusion coefficient is required.

Alongside to the net-peak potential, the half-peak width $\left(\Delta E_{\mathrm{p} / 2}\right)$ of the simulated SW voltammograms is also a parameter sensitive to the rate of the catalytic reaction. Fig. 5 shows the theoretical dependences of $\Delta E_{\mathrm{p} / 2}$ vs. $\log (\boldsymbol{z})$ obtained for three different values of $\log (K)$. The half-peak width changes from $130 \mathrm{mV}$ to a limiting value of $210 \mathrm{mV}$ under the influence of the catalytic parameter $\boldsymbol{z}$. In some regions of $\log (z)$, depending on the value of $\log (K)$, there is almost a linear dependence between $\Delta E_{\mathrm{p} / 2}$ and $\log (\boldsymbol{z})$. Note that for the Nernstian (electrochemically reversible) EC' mechanism, the half-peak width of the net SW voltammograms is insensitive to the rate of the catalytic reaction [21].

\subsection{Surface EC' mechanism}

For the surface EC' mechanism, the dimensionless current under conditions of SWV is defined as $\Psi=I\left[n F S \Gamma^{*}(\mathrm{Ox}) f\right]^{-1}$. For this mechanism the electrode kinetic parameter is defined as $\omega=k_{\text {sur }} / f$, while the dimensionless catalytic parameter $z=k_{\mathrm{c}} / f$ is identical as for the diffusional mechanism.

The computed SW voltammograms of the surface EC' mechanism under the influence of catalytic parameter $\boldsymbol{z}$ exhibit similar features as those elaborated for the diffusional EC' mechanism. Fig. 6 depicts SW voltammograms of the surface confined EC' mechanism featuring moderate electrode reaction $(\log (\omega)=-0.4)$, simulated for several values of the catalytic parameter $\boldsymbol{z}$. As in the previous mechanism, the peak-potential and the half-peak width of the net SW voltammograms are affected by the value of the catalytic parameter $z$. The linear parts of the dependences $\Delta E_{\mathrm{p} / 2} \mathrm{vs}$. $\log (\boldsymbol{z})$, simulated for three different values of $\log (\omega)$, are presented in Fig. 7a. As for the case of diffusional EC' mechanism, the slope of the linear part of the dependences $\Delta E_{\mathrm{p} / 2} v s . \log (z)$ is $-59 / n \mathrm{mV}$, while the intercept is defined as: intercept $=E^{0}+\mathbf{2 . 3 0 3 R T ( \alpha n F ) ^ { - 1 }}$ $\log (\omega)$. Depending on the value of the electrode kinetic parameter $\omega$, the half-peak width of the net SW voltammograms varies between 130 and $210 \mathrm{mV}$ (Fig. 7b). Each function in Fig. 7b is associated with a linear part, whose magnitude depends on $\omega$.

As previously known, the remarkable feature of the surface catalytic mechanism exhibiting fast electrode reaction is the splitting of the net SW voltammetric peak [1]. This phenomenon emerges

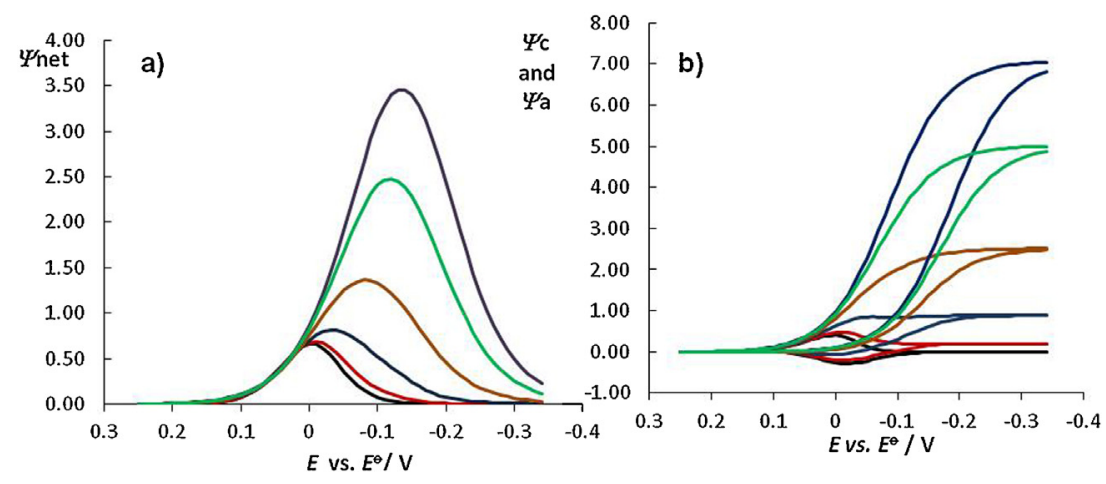

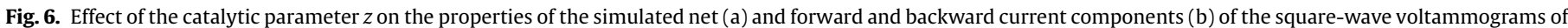

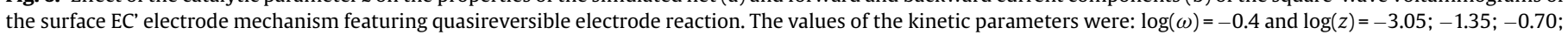
$-0.05 ; 0.40 ; 0.85$ (from lowest to the highest curve). The SW amplitude was $E_{\mathrm{sw}}=50 \mathrm{mV}$, and all other simulation parameters were the same as those in Fig. 2 . 

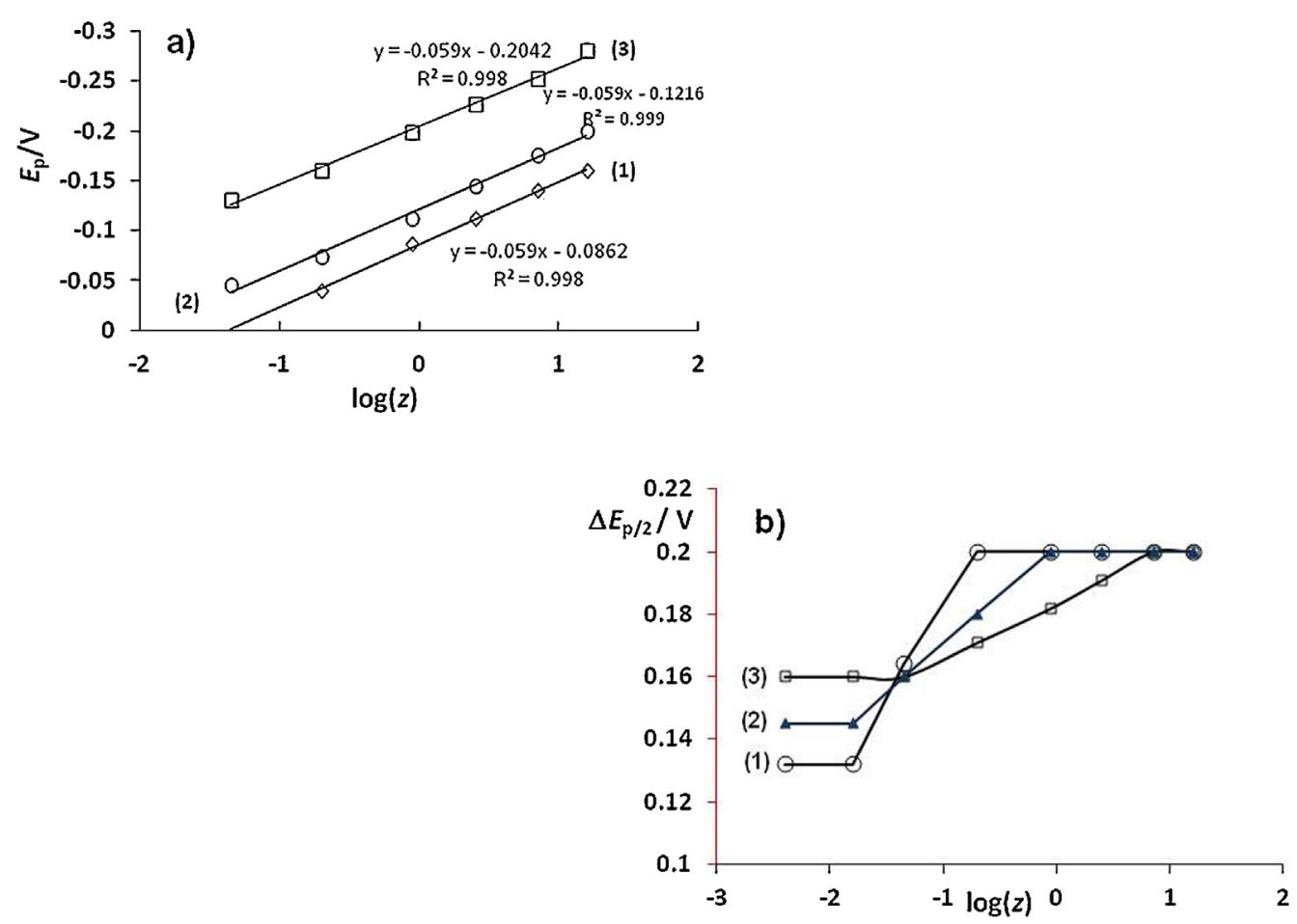

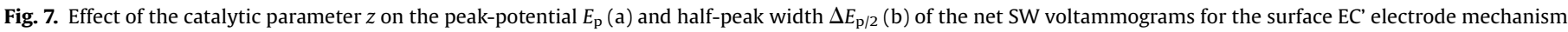

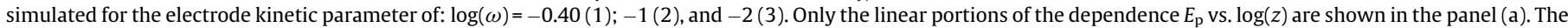
$\mathrm{SW}$ amplitude was $E_{\mathrm{sw}}=50 \mathrm{mV}$ and all other simulation parameters were the same as those in Fig. 2 .

either by the influence of the increased square-wave amplitude, or by the enhancement of the electrode kinetic parameter $\omega[1,8,16]$. The reasons for the splitting phenomenon are related to the potential dependence of the forward and backward rate constants of the electron transfer steps, and the specific chronoamperometric features of the surface redox reaction [1]. The critical values of the dimensionless kinetic parameter $\omega$ causing splitting of the net SWV peak depend on the number of exchanged electrons, the SW amplitude, and the cathodic electron transfer coefficient. This phenomenon occurs when the SW amplitude is higher than $25 \mathrm{mV}$, and the value of the dimensionless kinetic parameter $\omega$ is higher than 1.0 [1]. Fig. 8 shows several SW voltammograms of a fast electrode reaction that are simulated for several values of the catalytic parameter $\boldsymbol{z}$. As the catalytic parameter gets bigger values, the splitting of the SW net-peak phenomenon vanishes. That is because of the diminishing of the backward (re-oxidation) component of the square-wave voltammograms. This feature is caused by the chemical conversion of Red to Ox during the timeframe of the current measurements, under the influence of high values of the catalytic parameter $\boldsymbol{z}$. This phenomenon is exclusively
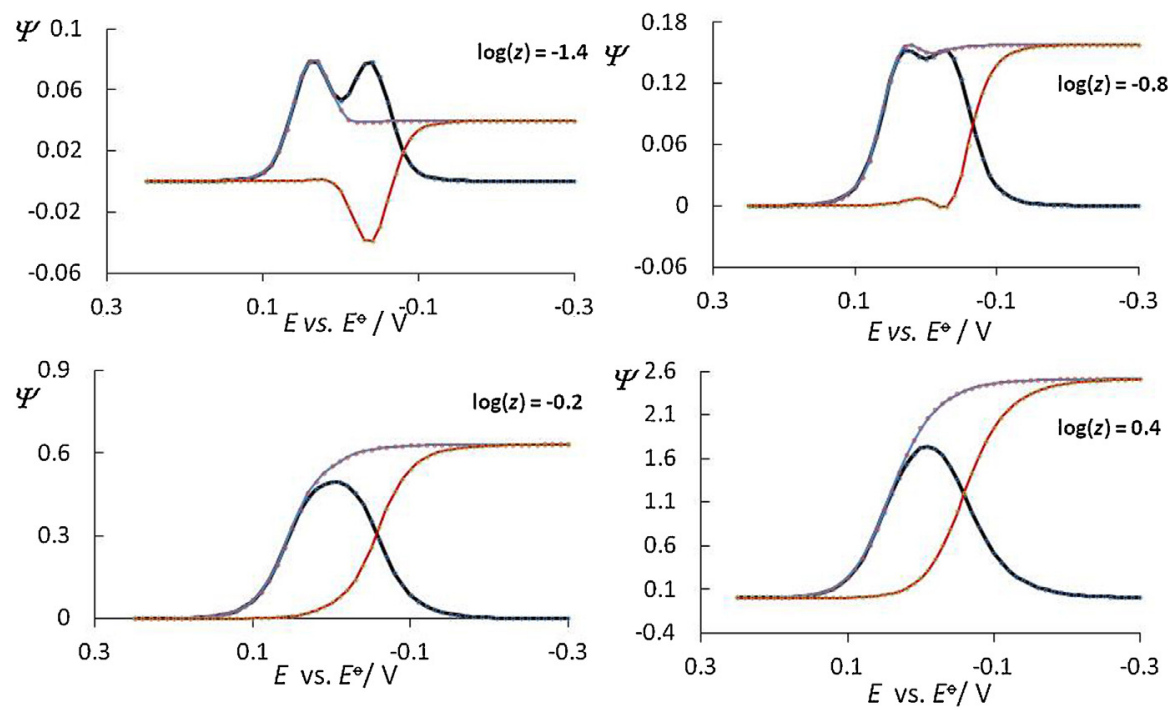

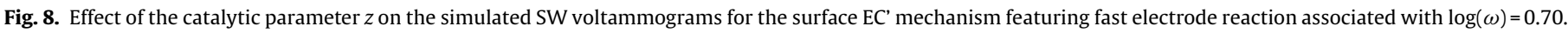
The SW amplitude was $E_{\mathrm{sw}}=50 \mathrm{mV}$ and all other simulation parameters were the same as those in Fig. 2. 
present by the surface electrode mechanism in SWV, and it distinguishes easily the surface EC' from the other surface redox mechanisms. It is worth mentioning that the splitting SW-peak phenomenon can be avoided either by using high values of the SW frequency, or small values of the SW amplitude. This, in turn, will allow the methods elaborated in this work (Fig. 7a) to be applied. More relevant features of the surface EC' mechanisms under conditions of SWV are elaborated elsewhere $[1,16,17]$.

\section{Conclusions}

During the last 30 years voltammetry has emerged as a powerful technique for getting insights into various redox mechanisms. It is also seen as a reliable tool for the determination of kinetics and thermodynamic parameters relevant to the electron transfer reactions and to the chemical reactions associated with the electrode reactions. All voltammetric methods developed for the kinetic and thermodynamic measurements elaborated so far rely either on the time-dependent [1-11] or potential-dependent measurements $[36,37]$. In this work we propose a new methodology to determine the kinetic parameters of the electrode reaction of an EC' mechanism from measurements in which the concentration of the regenerative reagent will be the only variable. As we have shown in Figs. 4 and 7a, a linear shift of the net SWV peak potentials of about $-59 \mathrm{mV} / \mathrm{n}$ is observed for every ten-fold increase of the catalytic parameter $\boldsymbol{z}$ for both diffusional and surface confined EC' reaction mechanisms. This phenomenon is observed if the kinetics of the chemical regenerative reaction is much faster than the kinetics of the electrode reaction. In such scenario, the intercept of the linear function $E_{\mathrm{p}} v s . \log (\boldsymbol{z})$ is defined as: intercept $=E^{0}+2.303 R T(\alpha n F)^{-1} \log (K)$. If we recall that at the EC' mechanisms in SWV the catalytic parameter $\boldsymbol{z}$ is defined as $\boldsymbol{z}=k_{\mathrm{c}} \mid f$, and $k_{\mathrm{c}}=k_{\mathrm{cat}} c(\mathrm{Y})$, then one can define the catalytic parameter as $\boldsymbol{z}=k_{\text {cat }} c(\mathrm{Y}) / f$. Therefore, the dependencies shown in Figs. 4 and $7 \mathrm{a}$ can be reproduced under constant frequency $f$, only by varying the concentration of the catalytic reagent Y. This, in turn, allows the standard rate constant of the electrode reaction ( $k_{\mathrm{s}}$ or $\left.k_{\mathrm{sur}}\right)$ to be determined from the intercept of the dependence $E_{\mathrm{p}} v s . \log [c(\mathrm{Y})]$. To determine the values of $k_{\mathrm{s}}$ or $k_{\text {sur }}$, one needs the values of the real chemical rate constant $k_{\text {cat }}$, electron transfer coefficient $\alpha$, the standard redox potential $E^{0}$, and the diffusion coefficient $D$ (the latter parameter only for the diffusional EC' mechanism). The methodologies to determine $k_{\text {cat }}, \alpha, E^{0}$, and $D$ can be found elsewhere $[1,9,10,16]$. The linear parts of the $E_{\mathrm{p}} \mathrm{vs} . \log (\boldsymbol{z})$ dependences depicted in Figs. 4 and 7a commonly hold true (approximately) if the ratio $\log \left[k_{\text {cat }} c(Y) f^{-1}\right] / \log \left[k_{\mathrm{s}} f^{-0.5} D^{-0.5}\right]>1$. For example, if we consider the case of the catalytic reaction of $\mathrm{Ti}^{3+}$ with $\mathrm{ClO}_{3}{ }^{-}$in acidic media, it is known from the literature that the catalytic rate constant $k_{\text {cat }}$ is about $155 \mathrm{~mol}^{-1} \mathrm{~L} \mathrm{~s}^{-1}$ [1]. The value of $k_{\mathrm{s}}$ for the redox reaction $\mathrm{Ti}^{4+} / \mathrm{Ti}^{3+}$ at the mercury working electrode in acidic media is about $9 \times 10^{-3} \mathrm{~cm} \mathrm{~s}^{-1}$ [38]. For the value of the diffusion coefficient $D$ of $\mathrm{Ti}^{4+}$ of $5 \times 10^{-6} \mathrm{~cm}^{2} \mathrm{~s}^{-1}$ and SW frequency of $10 \mathrm{~s}^{-1}$, the condition $\log \left[k_{\mathrm{cat}} c\left(\mathrm{ClO}_{3}{ }^{-}\right) f^{-1}\right] /\left[k_{\mathrm{s}} f^{-0.5} D^{-0.5}\right]>1$ will hold true for the concentration of $\mathrm{ClO}_{3}{ }^{-}$(the catalytic reagent) higher than $0.1 \mathrm{~mol} / \mathrm{L}$. Together with the temperature-related method for the determination of the kinetics of the electrode reaction reported in [39], this is another methodology for determination of the electrode kinetics, which is not time or potential dependent.

\section{Acknowledgments}

Both authors acknowledge Alexander von Humboldt foundation for the financial support from the Research Group Linkage Programme 3.4-Fokoop-DEU/1128670, as well as the support of DAAD foundation through multilateral project "International Masters and Postgraduate Programme in Materials Science and Catalysis" (MatCatNet). RG also acknowledges the Goce Delcev University-Stip, Macedonia, for providing funds via the University project.

\section{References}

[1] V. Mirceski, S. Komorsky-Lovric, M. Lovric, Square-Wave Voltammetry, Theory and Application, Springer, Berlin, 2007.

[2] A.J. Bard, L.R. Faulkner, Electrochemical Methods, Fundamentals and Applications, John Wiley \& Sons, New York, 2001.

[3] R.J. Dwayne Miller, G. McLendon, A.J. Nozik, A. Schmickler, F. Willig, Surface Electron Transfer Processes, Wiley, New York, 1995.

[4] M.R. Smyth, J.G. Vos, Analytical Voltammetry in Comprehensive Analytical Chemistry, Elsevier, New York, 1992.

[5] C.H. Bamfor, R.G. Compton, Electrode kinetics principles and methodology, Elsevier, New York, 1986.

[6] R.G. Compton, C.E. Banks, Understanding Voltammetry, World Scientific Publishing Co., Singapore, 2010.

[7] V. Mirceski, R. Gulaboski, M. Lovrić, I. Bogeski, R. Kappl, Reinhard. M. Hoth, Electroanal. 25 (2013) 2411.

[8] R. Gulaboski, V. Mirceski, I. Bogeski, M. Hoth, J. Solid State Electrochem. 16 (2012) 2315.

[9] R.S. Nicholson, Anal. Chem. 37 (1965) 1351.

[10] F. Garay, M. Lovric, J. Electroanal. Chem. 518 (2002) 91.

[11] J.J. O'Dea, J. Osteryoung, R.A. Osteryoung, Anal. Chem. 53 (1981) 695.

[12] D.H. Evans, Acc. Chem. Res. 10 (1977) 313.

[13] R. Gulaboski, P. Kokoskarova, S. Mitrev, Electrochim. Acta 69 (2012) 86

[14] R. Gulaboski, L. Mihajlov, Biophys. Chem. 155 (2011) 1.

[15] R. Gulaboski, J. Solid State Electrochem. 13 (2009) 1015.

[16] V. Mirceski, R. Gulaboski, J. Solid State Electrochem. 7 (2003) 157.

[17] V. Mirceski, R. Gulaboski, Electroanal. 13 (2001) 1326.

[18] J.M. Saveant, Elements of molecular and biomolecular electrochemistry, Wiley-Interscience, 2006.

[19] B.A. Brookes, J.C. Ball, R.G. Compton, J. Phys. Chem B 103 (1999) 5289.

[20] A. Molina, C. Serna, F. Martı'nez-Ortiz, J. Electroanal Chem 486 (2000) 9.

[21] A. Molina, J. Gonzalez, E. Laborda, Y. Wang, R.G. Compton, Phys. Chem. Chem. Phys. 13 (2011) 14694

[22] J. Galceran, S.L. Taylor, P.N. Bartlett, J. Electroanal. Chem. 466 (1999) 15-25.

[23] J. Galceran, S.L. Taylor, P.N. Bartlett, J. Electroanal. Chem. 476 (1999) 132

[24] Z. Galus, Fundamentals of electrochemical analysis, Halsted Press, Chichester, Ellis Horwood, New York, 1976.

[25] J.M. Saveant, Elements of molecular and biomolecular electrochemistry, Wiley-Interscience, 2006.

[26] J. González, C.M. Soto, A. Molina, Electrochim Acta 54 (2009) 6154

[27] Y. Shao, J. Wang, H. Wu, J. Liu, I.A. Aksay, Y. Lin, Electroanal. 22 (2010) 1027.

[28] A. Gass, G. Davis, G.D. Francis, H.O.A. Hill, W.J. Aston, I.J. Higgins, E.V. Plotkin, L. D.L. Scott, A.P.F. Turner, Anal. Chem. 56 (1984) 667.

[29] L. Gorton, Biosensors and modern biospecific analytical techniques, Elsevier, Amsterdam, 2005.

[30] G. Ramsay, Commercial biosensors, John Willey, New York, 1998.

[31] A. Molina, M.M. Moreno, C. Serna, F. Martinez-Ortiz, Electroanal.15 (2003) 254.

[32] D. Krulic, N. Larabi, N. Fatouros, J. Electroanal. Chem. 579 (2005) 243.

[33] A. Molina, J. Gonzalez, E. Laborda, Y. Wang, R.G. Compton, Phys. Chem. Chem. Phys. 13 (2011) 16748.

[34] J. Gonzalez, C.M. Soto, A. Molina, J. Electroanal. Chem. 634 (2009) 90.

[35] J. Heinze, Angew. Chemie 23 (1984) 831.

[36] A.J. Bard, G. Inzelt, F. Scholz, Electrochemical Dictionary, 2nd edition, Springer, 2012.

[37] V. Mirceski, E. Laborda, D. Guziejewsk, R.G. Compton, Anal. Chem. 85 (2013) 5586.

[38] M. Noel, P.N. Anantharaman, B. Electrochem. 3 (1987) 349.

[39] R. Gulaboski, M. Lovric, V. Mirceski, I. Bogeski, M. Hoth, Biophys. Chem. 137 (2008) 49. 